Acta Crystallographica Section E

Structure Reports

Online

ISSN 1600-5368

\section{1-Carboxymethyl-1'-carboxylatomethyl- $3,3^{\prime}$-[p-phenylenebis(oxymethylene)]- dipyridinium bromide dihydrate}

\section{Hong-Lei Lian ${ }^{a *}$ and Wei-Cheng Pan ${ }^{b}$}

${ }^{a}$ College of Chemical Engineering, Zhengzhou University, Zhengzhou, Henan 450001, People's Republic of China, and ' ${ }^{\mathbf{b}}$ College of Chemical Engineering and Foods, Zhongzhou University, Zhengzhou, Henan 450044, People's Republic of China

Correspondence e-mail: zzulhl@yahoo.com.cn

Received 12 August 2010; accepted 21 September 2010

Key indicators: single-crystal X-ray study; $T=293 \mathrm{~K}$; mean $\sigma(\mathrm{C}-\mathrm{C})=0.005 \AA$; $R$ factor $=0.045 ; w R$ factor $=0.090 ;$ data-to-parameter ratio $=13.4$.

In the crystal structure of the title salt, $\mathrm{C}_{22} \mathrm{H}_{21} \mathrm{~N}_{2} \mathrm{O}_{6}{ }^{+} \cdot-$ $\mathrm{Br}^{-} \cdot 2 \mathrm{H}_{2} \mathrm{O}$, pairs of betaine molecules are bridged by protons (the bridging proton is disordered), forming strong and symmetrical $\mathrm{O}-\mathrm{H} \cdots \mathrm{O}$ hydrogen bonds, leading to an infinite chain along the $b$ axis. The water molecules are linked to the betaine molecule and the bromide ion through $\mathrm{O}-\mathrm{H} \cdots \mathrm{O}$ and $\mathrm{O}-\mathrm{H} \cdots \mathrm{Br}$ interactions. The central ring, located on an inversion centre, makes dihedral angles of $1.2(2)^{\circ}$ with the outer rings. One of the carboxylic acid groups is deprotonated.

\section{Related literature}

For a related structure, see: Zhang et al. (2004).

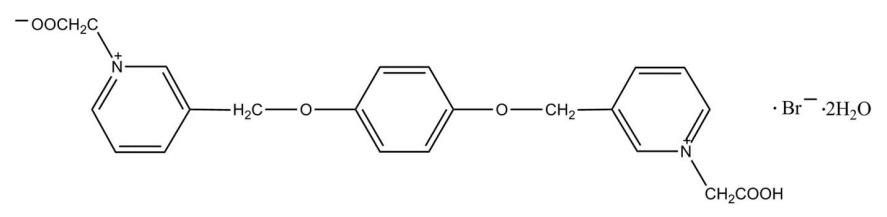

\section{Experimental}

Crystal data

$\mathrm{C}_{22} \mathrm{H}_{21} \mathrm{~N}_{2} \mathrm{O}_{6}{ }^{+} \cdot \mathrm{Br}^{-} \cdot 2 \mathrm{H}_{2} \mathrm{O}$

$M_{r}=525.35$
Monoclinic, $C 2 / c$

$a=20.605$ (4) А

$b=7.9612(12) \AA$

$c=15.233$ (4) $\AA$

$\beta=113.845(16)^{\circ}$

$V=2285.6(8) \AA^{3}$

Data collection

Bruker SMART CCD area-detector diffractometer

Absorption correction: multi-scan (SADABS; Sheldrick, 2004)

$T_{\min }=0.464, T_{\max }=0.556$

Refinement

$R\left[F^{2}>2 \sigma\left(F^{2}\right)\right]=0.045$

$w R\left(F^{2}\right)=0.090$

$S=1.09$

2009 reflections

$Z=4$

Mo $K \alpha$ radiation

$\mu=1.85 \mathrm{~mm}^{-1}$

$T=293 \mathrm{~K}$

$0.49 \times 0.43 \times 0.36 \mathrm{~mm}$

2537 measured reflections 2009 independent reflections 1520 reflections with $I>2 \sigma(I)$

$R_{\text {int }}=0.047$

Table 1

Hydrogen-bond geometry $\left(\AA,{ }^{\circ}\right)$.

\begin{tabular}{lllll}
\hline$D-\mathrm{H} \cdots A$ & $D-\mathrm{H}$ & $\mathrm{H} \cdots A$ & $D \cdots A$ & $D-\mathrm{H} \cdots A$ \\
\hline $\mathrm{O} 1-\mathrm{H} 1 \cdots \mathrm{O} 1^{\mathrm{i}}$ & 0.82 & 1.65 & $2.459(5)$ & 168 \\
$\mathrm{O} 4-\mathrm{H} 4 B \cdots \mathrm{Br} 1$ & 0.85 & 2.72 & $3.496(3)$ & 152 \\
$\mathrm{O} 4-\mathrm{H} 4 C \cdots \mathrm{O} 2^{\mathrm{ii}}$ & 0.85 & 2.25 & $3.040(4)$ & 155 \\
\hline
\end{tabular}

Symmetry codes: (i) $-x+\frac{1}{2},-y-\frac{1}{2},-z+1$; (ii) $-x+\frac{1}{2}, y+\frac{1}{2},-z+\frac{1}{2}$.

Data collection: SMART (Bruker, 2001); cell refinement: SAINT (Bruker, 2001); data reduction: $S A I N T$; $\operatorname{program}(\mathrm{s})$ used to solve structure: SHELXS97 (Sheldrick, 2008); program(s) used to refine structure: SHELXL97 (Sheldrick, 2008); molecular graphics: SHELXTL (Sheldrick, 2008); software used to prepare material for publication: SHELXTL and local programs.

Financial support from Zhengzhou University is greatly appreciated.

Supplementary data and figures for this paper are available from the IUCr electronic archives (Reference: PB2040).

\section{References}

Bruker (2001). SMART and SAINT. Bruker AXS Inc., Madison, Wisconsin, USA.

Sheldrick, G. M. (2004). SADABS. University of Göttingen, Germany.

Sheldrick, G. M. (2008). Acta Cryst. A64, 112-122.

Zhang, L.-P., Lam, C.-K., Song, H.-B. \& Mak, T. C. W. (2004). Polyhedron, 23, 2413-2425. 


\section{supporting information}

Acta Cryst. (2010). E66, o2656 [doi:10.1107/S1600536810037748]

\section{1-Carboxymethyl-1'-carboxylatomethyl-3,3'-[p-phenylenebis(oxymethyl- ene)]dipyridinium bromide dihydrate}

\section{Hong-Lei Lian and Wei-Cheng Pan}

\section{S1. Comment}

The design and synthesis of substrates for the ultimate preparation of supramolecular species has received much attention in recent years. Double betaines are a class of zwitterionic compounds possessing pairs of carboxylate groups and quaternary ammonium or pyridinium moieties. The carboxylate group is basic, so betaines are good proton acceptors that easily form complexes with Bronsted acids.

The synthesis and crystal structure of 1:2 salt of 1,4-bis(3-picolyloxyl)benzene- $N, N^{\prime}-$ diacetic acid with $\mathrm{HBr}$ has been reported, here we will describe the preparation and structure of the 1:1 salt.

In the crystal structure of the title compound, the phenylene ring of the title double betaine is located at an inversion center, making a dihedra angle of 1.2 degree. Pairs of the betaine molecules are bridged by protons to form strong and symmetrical $\mathrm{O} \cdots \mathrm{O}$ hydrogen bonds, leading to an infinite chain. The bromide ion is connected to the betaine molecule

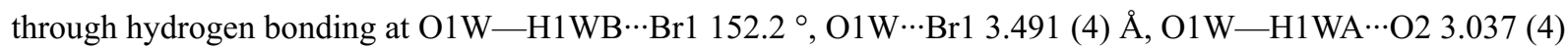
$\AA, \mathrm{O} 1 \mathrm{~W} \cdots \mathrm{O} 22154.5^{\circ}$ (Fig. 1).

\section{S2. Experimental}

1,4-bis(3-Picolyloxyl)benzene (2.92 g, $10 \mathrm{mmol})$ was dissolved in methanol (30 ml) to give a light yellow solution, to which ethyl bromoacetate ( $3 \mathrm{ml}, 27 \mathrm{mmol}$, Aldrich) was added. The resulting solution was refluxed for 3 days. After the methanol was removed by rotary evaporation under reduced pressure, hydrobromic acid $(12.5 \mathrm{ml}, 4.8 \%(w / v))$ was added to the yellow residue. The mixture was refluxed for $24 \mathrm{~h}$ to give a yellow solution. Removal of solvent afforded a light yellow powdery product. Yield: $46 \%$. It was re-crystallized in water to obtain suitable single crystals for X-ray analysis.

\section{S3. Refinement}

$\mathrm{H}$ atoms in water molecule were located in a difference map. Other $\mathrm{H}$ atoms were positioned geometrically and refined using a riding model with $\mathrm{C}-\mathrm{H}=0.95-0.99 \AA$ and with $U_{\text {iso }}(\mathrm{H})=1.2 U_{\mathrm{eq}}(\mathrm{C})$. 


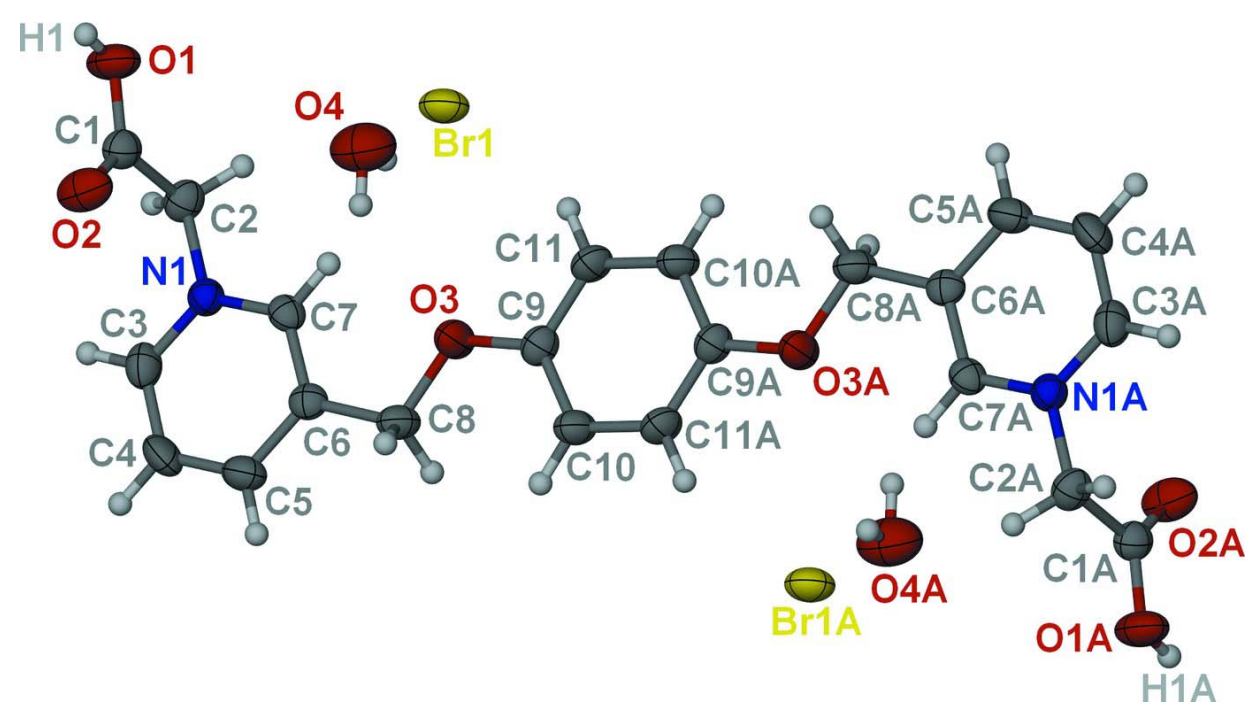

\section{Figure 1}

Ellipsoid plot.

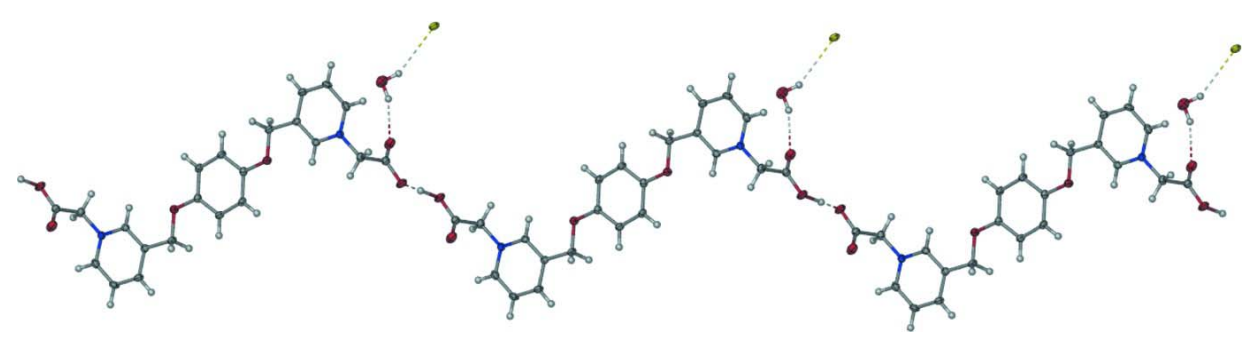

\section{Figure 2}

A portion of the infinite chain of the title compound viewed along the a direction, with atom labels of $30 \%$ probability displacement ellipsoids. Hydrogen bonds are displayed with dashed lines.

\section{1-Carboxymethyl-1'-carboxylatomethyl-3,3'-[p- phenylenebis(oxymethylene)]dipyridinium bromide dihydrate}

\section{Crystal data}

$\mathrm{C}_{22} \mathrm{H}_{21} \mathrm{~N}_{2} \mathrm{O}_{6}{ }^{+} \cdot \mathrm{Br}^{-} \cdot 2 \mathrm{H}_{2} \mathrm{O}$

$M_{r}=525.35$

Monoclinic, $C 2 / c$

$a=20.605$ (4) $\AA$

$b=7.9612(12) \AA$

$c=15.233$ (4) $\AA$

$\beta=113.845(16)^{\circ}$

$V=2285.6(8) \AA^{3}$

$Z=4$

\section{Data collection}

Bruker SMART CCD area-detector diffractometer

Radiation source: fine-focus sealed tube Graphite monochromator phi and $\omega$ scans
$F(000)=1080$

$D_{\mathrm{x}}=1.527 \mathrm{Mg} \mathrm{m}^{-3}$

Mo $K \alpha$ radiation, $\lambda=0.71073 \AA$

Cell parameters from 186 reflections

$\theta=2.0-27.6^{\circ}$

$\mu=1.85 \mathrm{~mm}^{-1}$

$T=293 \mathrm{~K}$

Block, light yellow

$0.49 \times 0.43 \times 0.36 \mathrm{~mm}$ 


$$
\begin{aligned}
& R_{\text {int }}=0.047 \\
& \theta_{\max }=25.0^{\circ}, \theta_{\min }=2.2^{\circ} \\
& h=-1 \rightarrow 24
\end{aligned}
$$

\section{Refinement}

Refinement on $F^{2}$

Least-squares matrix: full

$R\left[F^{2}>2 \sigma\left(F^{2}\right)\right]=0.045$

$w R\left(F^{2}\right)=0.090$

$S=1.09$

2009 reflections

150 parameters

0 restraints

Primary atom site location: structure-invariant direct methods $k=-1 \rightarrow 9$

$l=-18 \rightarrow 16$

Secondary atom site location: difference Fourier map

Hydrogen site location: inferred from neighbouring sites

$\mathrm{H}$-atom parameters constrained

$w=1 /\left[\sigma^{2}\left(F_{\mathrm{o}}^{2}\right)+(0.0331 P)^{2}+1.7877 P\right]$

where $P=\left(F_{\mathrm{o}}^{2}+2 F_{\mathrm{c}}^{2}\right) / 3$

$(\Delta / \sigma)_{\max }<0.001$

$\Delta \rho_{\max }=0.52 \mathrm{e} \AA^{-3}$

$\Delta \rho_{\min }=-0.33$ e $\AA^{-3}$

Special details

Geometry. All e.s.d.'s (except the e.s.d. in the dihedral angle between two 1.s. planes) are estimated using the full covariance matrix. The cell e.s.d.'s are taken into account individually in the estimation of e.s.d.'s in distances, angles and torsion angles; correlations between e.s.d.'s in cell parameters are only used when they are defined by crystal symmetry. An approximate (isotropic) treatment of cell e.s.d.'s is used for estimating e.s.d.'s involving 1.s. planes.

Refinement. Refinement of $F^{2}$ against ALL reflections. The weighted $R$-factor $w R$ and goodness of fit $S$ are based on $F^{2}$, conventional $R$-factors $R$ are based on $F$, with $F$ set to zero for negative $F^{2}$. The threshold expression of $F^{2}>\sigma\left(F^{2}\right)$ is used only for calculating $R$-factors $(\mathrm{gt})$ etc. and is not relevant to the choice of reflections for refinement. $R$-factors based on $F^{2}$

\begin{tabular}{|c|c|c|c|c|c|}
\hline & $x$ & $y$ & $z$ & $U_{\text {iso }} * / U_{\text {eq }}$ & Occ. $(<1)$ \\
\hline $\mathrm{Br} 1$ & 0.0000 & $0.04115(8)$ & 0.2500 & $0.0454(2)$ & \\
\hline $\mathrm{O} 1$ & $0.20052(13)$ & $-0.2950(4)$ & $0.42592(16)$ & $0.0527(7)$ & \\
\hline H1 & 0.2364 & -0.2608 & 0.4703 & $0.079^{*}$ & 0.50 \\
\hline $\mathrm{O} 2$ & $0.26011(13)$ & -0.2308 (4) & $0.33644(16)$ & $0.0519(7)$ & \\
\hline $\mathrm{O} 3$ & $0.05771(12)$ & $0.1842(3)$ & $0.05634(16)$ & $0.0410(6)$ & \\
\hline N1 & 0.14157 (13) & $-0.2645(3)$ & $0.16985(17)$ & $0.0307(6)$ & \\
\hline $\mathrm{C} 1$ & 0.20678 (19) & $-0.2737(4)$ & $0.3465(2)$ & $0.0351(8)$ & \\
\hline $\mathrm{C} 2$ & $0.13832(18)$ & $-0.3130(5)$ & $0.2610(2)$ & $0.0366(8)$ & \\
\hline $\mathrm{H} 2 \mathrm{~A}$ & 0.1288 & -0.4324 & 0.2600 & $0.044^{*}$ & \\
\hline $\mathrm{H} 2 \mathrm{~B}$ & 0.0995 & -0.2538 & 0.2679 & $0.044^{*}$ & \\
\hline $\mathrm{C} 3$ & $0.17237(18)$ & $-0.3677(5)$ & $0.1291(2)$ & $0.0398(9)$ & \\
\hline $\mathrm{H} 3 \mathrm{~A}$ & 0.1902 & -0.4708 & 0.1572 & $0.048^{*}$ & \\
\hline $\mathrm{C} 4$ & $0.17744(18)$ & $-0.3203(5)$ & $0.0454(3)$ & $0.0454(10)$ & \\
\hline $\mathrm{H} 4 \mathrm{~A}$ & 0.1982 & -0.3922 & 0.0161 & $0.054^{*}$ & \\
\hline $\mathrm{C} 5$ & $0.15208(18)$ & $-0.1677(5)$ & $0.0053(2)$ & $0.0410(9)$ & \\
\hline H5A & 0.1561 & -0.1351 & -0.0509 & $0.049 *$ & \\
\hline C6 & $0.12032(16)$ & -0.0615 (4) & $0.0482(2)$ & $0.0304(8)$ & \\
\hline $\mathrm{C} 7$ & $0.11602(16)$ & $-0.1142(4)$ & $0.1314(2)$ & $0.0303(8)$ & \\
\hline $\mathrm{H} 7 \mathrm{~A}$ & 0.0951 & -0.0448 & 0.1617 & $0.036^{*}$ & \\
\hline $\mathrm{C} 8$ & 0.09107 (19) & 0.1032 & $0.0026(2)$ & $0.0367(8)$ & \\
\hline H8A & 0.1291 & 0.1732 & 0.0008 & $0.044^{*}$ & \\
\hline H8B & 0.0569 & 0.0855 & -0.0628 & $0.044 *$ & \\
\hline
\end{tabular}
are statistically about twice as large as those based on $F$, and $R$ - factors based on ALL data will be even larger.

Fractional atomic coordinates and isotropic or equivalent isotropic displacement parameters $\left(\AA^{2}\right)$ 


\begin{tabular}{lllll} 
C9 & $0.02970(17)$ & $0.3417(4)$ & $0.0252(2)$ & $0.0327(8)$ \\
C10 & $0.02951(18)$ & $0.4206(4)$ & $-0.0558(2)$ & $0.0368(9)$ \\
H10A & 0.0492 & 0.3675 & -0.0937 & $0.044^{*}$ \\
C11 & $-0.00005(18)$ & $0.4215(4)$ & $0.0802(2)$ & $0.0367(9)$ \\
H11A & -0.0003 & 0.3684 & 0.1344 & $0.044^{*}$ \\
O4 & $0.15569(16)$ & $0.2619(4)$ & $0.28766(19)$ & $0.0738(9)$ \\
H4B & 0.1174 & 0.2082 & 0.2578 & $0.111^{*}$ \\
H4C & 0.1662 & 0.2720 & 0.2395 & $0.111^{*}$ \\
\hline
\end{tabular}

Atomic displacement parameters $\left(\AA^{2}\right)$

\begin{tabular}{lllllll}
\hline & $U^{11}$ & $U^{22}$ & $U^{33}$ & $U^{12}$ & $U^{13}$ & $U^{23}$ \\
\hline $\mathrm{Br} 1$ & $0.0537(4)$ & $0.0565(4)$ & $0.0345(3)$ & 0.000 & $0.0266(2)$ & 0.000 \\
$\mathrm{O} 1$ & $0.0496(16)$ & $0.081(2)$ & $0.0317(13)$ & $-0.0026(15)$ & $0.0210(12)$ & $-0.0007(13)$ \\
$\mathrm{O} 2$ & $0.0408(15)$ & $0.075(2)$ & $0.0394(14)$ & $-0.0151(14)$ & $0.0154(12)$ & $0.0005(13)$ \\
$\mathrm{O} 3$ & $0.0565(16)$ & $0.0336(15)$ & $0.0416(13)$ & $0.0120(12)$ & $0.0289(12)$ & $0.0107(11)$ \\
$\mathrm{N} 1$ & $0.0294(15)$ & $0.0312(17)$ & $0.0302(14)$ & $0.0004(13)$ & $0.0108(12)$ & $-0.0002(13)$ \\
$\mathrm{C} 1$ & $0.039(2)$ & $0.032(2)$ & $0.0343(18)$ & $0.0022(17)$ & $0.0150(16)$ & $0.0015(16)$ \\
$\mathrm{C} 2$ & $0.037(2)$ & $0.035(2)$ & $0.0370(19)$ & $-0.0008(17)$ & $0.0145(16)$ & $0.0067(16)$ \\
$\mathrm{C} 3$ & $0.038(2)$ & $0.033(2)$ & $0.043(2)$ & $0.0067(17)$ & $0.0109(17)$ & $0.0006(18)$ \\
$\mathrm{C} 4$ & $0.045(2)$ & $0.049(3)$ & $0.045(2)$ & $0.014(2)$ & $0.0218(19)$ & $-0.009(2)$ \\
$\mathrm{C} 5$ & $0.044(2)$ & $0.051(3)$ & $0.0335(18)$ & $0.0026(19)$ & $0.0206(17)$ & $-0.0030(18)$ \\
$\mathrm{C} 6$ & $0.0294(17)$ & $0.032(2)$ & $0.0280(16)$ & $-0.0034(16)$ & $0.0100(14)$ & $-0.0026(16)$ \\
$\mathrm{C} 7$ & $0.0305(18)$ & $0.0292(19)$ & $0.0310(16)$ & $-0.0007(16)$ & $0.0122(15)$ & $-0.0046(15)$ \\
$\mathrm{C} 8$ & $0.048(2)$ & $0.037(2)$ & $0.0305(17)$ & $0.0007(18)$ & $0.0209(16)$ & $0.0008(16)$ \\
$\mathrm{C} 9$ & $0.0351(19)$ & $0.029(2)$ & $0.0335(17)$ & $-0.0007(17)$ & $0.0129(15)$ & $0.0052(16)$ \\
$\mathrm{C} 10$ & $0.048(2)$ & $0.036(2)$ & $0.0313(17)$ & $0.0031(17)$ & $0.0207(16)$ & $0.0016(16)$ \\
C11 & $0.046(2)$ & $0.036(2)$ & $0.0317(17)$ & $0.0028(17)$ & $0.0192(16)$ & $0.0097(15)$ \\
O4 & $0.076(2)$ & $0.094(2)$ & $0.0528(17)$ & $-0.0131(19)$ & $0.0270(16)$ & $-0.0071(17)$ \\
& & & & & & \\
\hline
\end{tabular}

Geometric parameters $\left(A,{ }^{\circ}\right)$

\begin{tabular}{llll}
\hline $\mathrm{Br} 1-\mathrm{Br} 1$ & $0.0000(12)$ & $\mathrm{C} 5-\mathrm{C} 6$ & $1.385(4)$ \\
$\mathrm{O} 1-\mathrm{C} 1$ & $1.279(4)$ & $\mathrm{C} 5-\mathrm{H} 5 \mathrm{~A}$ & 0.9300 \\
$\mathrm{O} 1-\mathrm{H} 1$ & 0.8200 & $\mathrm{C} 6-\mathrm{C} 7$ & $1.372(4)$ \\
$\mathrm{O} 2-\mathrm{C} 1$ & $1.218(4)$ & $\mathrm{C} 6-\mathrm{C} 8$ & $1.492(5)$ \\
$\mathrm{O} 3-\mathrm{C} 9$ & $1.382(4)$ & $\mathrm{C} 7-\mathrm{H} 7 \mathrm{~A}$ & 0.9300 \\
$\mathrm{O} 3-\mathrm{C} 8$ & $1.419(4)$ & $\mathrm{C} 8-\mathrm{H} 8 \mathrm{~A}$ & 0.9700 \\
$\mathrm{~N} 1-\mathrm{C} 3$ & $1.335(4)$ & $\mathrm{C} 8-\mathrm{H} 8 \mathrm{~B}$ & 0.9700 \\
$\mathrm{~N} 1-\mathrm{C} 7$ & $1.342(4)$ & $\mathrm{C} 9-\mathrm{C} 11$ & $1.376(4)$ \\
$\mathrm{N} 1-\mathrm{C} 2$ & $1.469(4)$ & $\mathrm{C} 9-\mathrm{C} 10$ & $1.383(4)$ \\
$\mathrm{C} 1-\mathrm{C} 2$ & $1.516(5)$ & $\mathrm{C} 10-\mathrm{C} 11^{\mathrm{i}}$ & $1.380(5)$ \\
$\mathrm{C} 2-\mathrm{H} 2 \mathrm{~A}$ & 0.9700 & $\mathrm{C} 10-\mathrm{H} 10 \mathrm{~A}$ & 0.9300 \\
$\mathrm{C} 2-\mathrm{H} 2 \mathrm{~B}$ & 0.9700 & $\mathrm{C} 11-\mathrm{C} 10^{\mathrm{i}}$ & $1.380(5)$ \\
$\mathrm{C} 3-\mathrm{C} 4$ & $1.373(5)$ & $\mathrm{C} 11-\mathrm{H} 11 \mathrm{~A}$ & 0.9300 \\
$\mathrm{C} 3-\mathrm{H} 3 \mathrm{~A}$ & 0.9300 & $\mathrm{O} 4-\mathrm{H} 4 \mathrm{~B}$ & 0.8498 \\
$\mathrm{C} 4-\mathrm{C} 5$ & $1.366(5)$ & $\mathrm{O} 4-\mathrm{H} 4 \mathrm{C}$ & 0.8494 \\
$\mathrm{C} 4-\mathrm{H} 4 \mathrm{~A}$ & 0.9300 & &
\end{tabular}




\begin{tabular}{|c|c|c|c|}
\hline $\mathrm{C} 1-\mathrm{O} 1-\mathrm{H} 1$ & 109.5 & $\mathrm{C} 7-\mathrm{C} 6-\mathrm{C} 5$ & $118.0(3)$ \\
\hline $\mathrm{C} 9-\mathrm{O} 3-\mathrm{C} 8$ & $116.5(2)$ & $\mathrm{C} 7-\mathrm{C} 6-\mathrm{C} 8$ & $122.3(3)$ \\
\hline $\mathrm{C} 3-\mathrm{N} 1-\mathrm{C} 7$ & $121.5(3)$ & $\mathrm{C} 5-\mathrm{C} 6-\mathrm{C} 8$ & $119.7(3)$ \\
\hline $\mathrm{C} 3-\mathrm{N} 1-\mathrm{C} 2$ & $119.3(3)$ & $\mathrm{N} 1-\mathrm{C} 7-\mathrm{C} 6$ & $121.0(3)$ \\
\hline $\mathrm{C} 7-\mathrm{N} 1-\mathrm{C} 2$ & $119.1(3)$ & $\mathrm{N} 1-\mathrm{C} 7-\mathrm{H} 7 \mathrm{~A}$ & 119.5 \\
\hline $\mathrm{O} 2-\mathrm{C} 1-\mathrm{O} 1$ & $126.7(3)$ & $\mathrm{C} 6-\mathrm{C} 7-\mathrm{H} 7 \mathrm{~A}$ & 119.5 \\
\hline $\mathrm{O} 2-\mathrm{C} 1-\mathrm{C} 2$ & $121.6(3)$ & $\mathrm{O} 3-\mathrm{C} 8-\mathrm{C} 6$ & $109.2(2)$ \\
\hline $\mathrm{O} 1-\mathrm{C} 1-\mathrm{C} 2$ & $111.7(3)$ & $\mathrm{O} 3-\mathrm{C} 8-\mathrm{H} 8 \mathrm{~A}$ & 109.8 \\
\hline $\mathrm{N} 1-\mathrm{C} 2-\mathrm{C} 1$ & $112.0(3)$ & $\mathrm{C} 6-\mathrm{C} 8-\mathrm{H} 8 \mathrm{~A}$ & 109.8 \\
\hline $\mathrm{N} 1-\mathrm{C} 2-\mathrm{H} 2 \mathrm{~A}$ & 109.2 & $\mathrm{O} 3-\mathrm{C} 8-\mathrm{H} 8 \mathrm{~B}$ & 109.8 \\
\hline $\mathrm{C} 1-\mathrm{C} 2-\mathrm{H} 2 \mathrm{~A}$ & 109.2 & $\mathrm{C} 6-\mathrm{C} 8-\mathrm{H} 8 \mathrm{~B}$ & 109.8 \\
\hline $\mathrm{N} 1-\mathrm{C} 2-\mathrm{H} 2 \mathrm{~B}$ & 109.2 & $\mathrm{H} 8 \mathrm{~A}-\mathrm{C} 8-\mathrm{H} 8 \mathrm{~B}$ & 108.3 \\
\hline $\mathrm{C} 1-\mathrm{C} 2-\mathrm{H} 2 \mathrm{~B}$ & 109.2 & $\mathrm{C} 11-\mathrm{C} 9-\mathrm{O} 3$ & $115.9(3)$ \\
\hline $\mathrm{H} 2 \mathrm{~A}-\mathrm{C} 2-\mathrm{H} 2 \mathrm{~B}$ & 107.9 & $\mathrm{C} 11-\mathrm{C} 9-\mathrm{C} 10$ & $119.4(3)$ \\
\hline $\mathrm{N} 1-\mathrm{C} 3-\mathrm{C} 4$ & $119.4(3)$ & $\mathrm{O} 3-\mathrm{C} 9-\mathrm{C} 10$ & $124.6(3)$ \\
\hline $\mathrm{N} 1-\mathrm{C} 3-\mathrm{H} 3 \mathrm{~A}$ & 120.3 & $\mathrm{C} 11^{\mathrm{i}}-\mathrm{C} 10-\mathrm{C} 9$ & $119.7(3)$ \\
\hline $\mathrm{C} 4-\mathrm{C} 3-\mathrm{H} 3 \mathrm{~A}$ & 120.3 & $\mathrm{C} 11^{\mathrm{i}}-\mathrm{C} 10-\mathrm{H} 10 \mathrm{~A}$ & 120.1 \\
\hline $\mathrm{C} 5-\mathrm{C} 4-\mathrm{C} 3$ & $120.1(3)$ & $\mathrm{C} 9-\mathrm{C} 10-\mathrm{H} 10 \mathrm{~A}$ & 120.1 \\
\hline $\mathrm{C} 5-\mathrm{C} 4-\mathrm{H} 4 \mathrm{~A}$ & 119.9 & $\mathrm{C} 9-\mathrm{C} 11-\mathrm{C} 10^{\mathrm{i}}$ & $120.8(3)$ \\
\hline $\mathrm{C} 3-\mathrm{C} 4-\mathrm{H} 4 \mathrm{~A}$ & 119.9 & $\mathrm{C} 9-\mathrm{C} 11-\mathrm{H} 11 \mathrm{~A}$ & 119.6 \\
\hline $\mathrm{C} 4-\mathrm{C} 5-\mathrm{C} 6$ & $120.0(3)$ & $\mathrm{C} 10^{\mathrm{i}}-\mathrm{C} 11-\mathrm{H} 11 \mathrm{~A}$ & 119.6 \\
\hline $\mathrm{C} 4-\mathrm{C} 5-\mathrm{H} 5 \mathrm{~A}$ & 120.0 & $\mathrm{H} 4 \mathrm{~B}-\mathrm{O} 4-\mathrm{H} 4 \mathrm{C}$ & 95.3 \\
\hline $\mathrm{C} 6-\mathrm{C} 5-\mathrm{H} 5 \mathrm{~A}$ & 120.0 & & \\
\hline $\mathrm{C} 3-\mathrm{N} 1-\mathrm{C} 2-\mathrm{C} 1$ & $82.2(4)$ & $\mathrm{C} 5-\mathrm{C} 6-\mathrm{C} 7-\mathrm{N} 1$ & $-0.3(5)$ \\
\hline $\mathrm{C} 7-\mathrm{N} 1-\mathrm{C} 2-\mathrm{C} 1$ & $-95.1(3)$ & $\mathrm{C} 8-\mathrm{C} 6-\mathrm{C} 7-\mathrm{N} 1$ & $178.7(3)$ \\
\hline $\mathrm{O} 2-\mathrm{C} 1-\mathrm{C} 2-\mathrm{N} 1$ & $-10.1(5)$ & $\mathrm{C} 9-\mathrm{O} 3-\mathrm{C} 8-\mathrm{C} 6$ & $177.9(3)$ \\
\hline $\mathrm{O} 1-\mathrm{C} 1-\mathrm{C} 2-\mathrm{N} 1$ & $171.3(3)$ & $\mathrm{C} 7-\mathrm{C} 6-\mathrm{C} 8-\mathrm{O} 3$ & $-2.4(4)$ \\
\hline $\mathrm{C} 7-\mathrm{N} 1-\mathrm{C} 3-\mathrm{C} 4$ & $-0.7(5)$ & $\mathrm{C} 5-\mathrm{C} 6-\mathrm{C} 8-\mathrm{O} 3$ & $176.5(3)$ \\
\hline $\mathrm{C} 2-\mathrm{N} 1-\mathrm{C} 3-\mathrm{C} 4$ & $-178.0(3)$ & $\mathrm{C} 8-\mathrm{O} 3-\mathrm{C} 9-\mathrm{C} 11$ & $-177.6(3)$ \\
\hline $\mathrm{N} 1-\mathrm{C} 3-\mathrm{C} 4-\mathrm{C} 5$ & $1.0(5)$ & $\mathrm{C} 8-\mathrm{O} 3-\mathrm{C} 9-\mathrm{C} 10$ & $2.8(5)$ \\
\hline $\mathrm{C} 3-\mathrm{C} 4-\mathrm{C} 5-\mathrm{C} 6$ & $-0.9(5)$ & $\mathrm{C} 11-\mathrm{C} 9-\mathrm{C} 10-\mathrm{C} 11^{\mathrm{i}}$ & $0.5(6)$ \\
\hline $\mathrm{C} 4-\mathrm{C} 5-\mathrm{C} 6-\mathrm{C} 7$ & $0.5(5)$ & $\mathrm{O} 3-\mathrm{C} 9-\mathrm{C} 10-\mathrm{C} 11^{\mathrm{i}}$ & $-180.0(3)$ \\
\hline $\mathrm{C} 4-\mathrm{C} 5-\mathrm{C} 6-\mathrm{C} 8$ & $-178.5(3)$ & $\mathrm{O} 3-\mathrm{C} 9-\mathrm{C} 11-\mathrm{C} 10^{\mathrm{i}}$ & $179.9(3)$ \\
\hline $\mathrm{C} 3-\mathrm{N} 1-\mathrm{C} 7-\mathrm{C} 6$ & $0.4(5)$ & $\mathrm{C} 10-\mathrm{C} 9-\mathrm{C} 11-\mathrm{C} 10^{\mathrm{i}}$ & $-0.5(6)$ \\
\hline $\mathrm{C} 2-\mathrm{N} 1-\mathrm{C} 7-\mathrm{C} 6$ & $177.6(3)$ & & \\
\hline
\end{tabular}

Symmetry code: (i) $-x,-y+1,-z$.

Hydrogen-bond geometry $\left(\AA,{ }^{\circ}\right)$

\begin{tabular}{lllll}
\hline$D-\mathrm{H} \cdots A$ & $D-\mathrm{H}$ & $\mathrm{H} \cdots A$ & $D \cdots A$ & $D-\mathrm{H} \cdots A$ \\
\hline $\mathrm{O} 1-\mathrm{H} 1 \cdots \mathrm{O} 1^{\mathrm{ii}}$ & 0.82 & 1.65 & $2.459(5)$ & 168 \\
$\mathrm{O} 4-\mathrm{H} 4 B \cdots \mathrm{Br} 1$ & 0.85 & 2.72 & $3.496(3)$ & 152 \\
$\mathrm{O} 4-\mathrm{H} 4 C \cdots \mathrm{O} 22^{\mathrm{iii}}$ & 0.85 & 2.25 & $3.040(4)$ & 155 \\
\hline
\end{tabular}

Symmetry codes: (ii) $-x+1 / 2,-y-1 / 2,-z+1$; (iii) $-x+1 / 2, y+1 / 2,-z+1 / 2$. 